\title{
Whither Commercial Nanobiosensors?
}

\section{Komandoor Achyuthan}

Sandia National Laboratories, Albuquerque, NM 87508, USA

The excitement surrounding the marriage of biosensors and nanotechnology is palpable even from a cursory examination of the scientific literature. Indeed, the word "nano" might be in danger of being overused and reduced to a cliché, although probably essential for publishing papers or securing research funding. The biosensor literature is littered with clever or catchy acronyms, birds being apparently favored ("CANARY", "SPARROW"), quite apart from "electronic tongue," "electronic nose," and so on. Although biosensors have been around since glucose monitors were commercialized in the 1970s, the transition of laboratory research and innumerable research papers on biosensors into the world of commerce has lagged. There are several reasons for this phenomenon including the infamous "valley of death" afflicting entrepreneurs emerging from academic environment into the industrial world, where the rules for success can be radically different.

In this context, musings on biosensors and especially nanobiosensors in an open access journal such as Journal of Biosensors and Bioelectronics is topical and appropriate especially since market surveys of biosensors are prohibitively expensive, sometimes running into thousands of dollars for a single copy. The contents and predictions of market share for biosensors in these reports also keep changing every time a report is published. Not only that, the market share projections for biosensors differs considerably amongst various reports. An editorial provides the opportunity to offer personal opinions and perhaps stimulate debate on a particular topic. In this sense, editorials are a departure from the rigor of a research paper. This editorial is no exception. With this preamble, it is worthwhile to stop and ponder the status of commercial biosensors and nanobiosensors.

A PubMed search for reviews on "commercial nanobiosensors" and "commercial biosensors" revealed 1 and 71 hits, respectively (accessed 2/12/2011). This may be due to commercial nanobiosensors lagging behind academic research and/or nanobiosensors not being ready for primetime in the marketplace. Are nanobiosensors largely lab curiosities or is there a near-term, cost-competitive, successful commercialization prospect? Despite decades of research and millions of dollars spent, one single analyte, namely glucose, dominates the commercial biosensor market share, even though heart disease, cancer and stroke are far more prevalent in the US relative to diabetes. This is a reflection of the formidable challenges confronting biosensors getting out of the lab and into the hands of the end-user, where entirely unanticipated situations arise. Such challenges will be magnified while commercializing nanobiosensors. In the crudest parlance, despite the boast of nanometer size range of nanobiosensor components, they must nevertheless be integrated within an enclosure or a framework that fits comfortably in a human hand with a display visible to the eyes, and be properly manipulated using fingers during actual use. Alternately, nanobiosensor elements may be interfaced with macro elements of "regular" biosensors, in which case the result is a hybrid device and not strictly a "nanobiosensor." Going "nano" globally, with portable fielddeployable nanobiosensors means a readiness for handling nanoliter volumes that can rapidly evaporate under uncontrolled "ambient temperatures" around the world, and contain fewer biomolecules to detect due to sample size limitation, all without sacrificing key assay metrics of sensitivity or specificity.
This transition from the nano- to the macro-world is a nontrivial task and only gets harder depending on the end-user's age, health, education, and overall comfort level with technology. If one argues that sophisticated machines capable of automatically handling nanobiosensors will obviate human intervention, then one must consider the costs associated with such machine handlers. In large swaths of the world, morbidity and mortality are on the rise from diseases such as influenza, cholera and polio and where people often suffer from malnutrition, hunger, poverty, famine and a lack of hygiene or potable water. Clearly these factors are rarely encountered in the western society where the bulk of nano-research is executed. Even in emerging economies there appears to be a focus on "nano" despite encountering the formidable challenges listed above. Given this situation, is there truly a need for expensive, sophisticated nanobiosensors?

Additional factors to consider on the topic of the commercialization of biosensors in general and nanobiosensors in particular, include making the technology transparent to the end-user and inquiring whether technological advances overlap the skills of the "average" user. Perhaps the lessons of corn-based ethanol and the unintended consequences of "food-versus-fuel" debate are obliquely relevant to nanobiosensors. In the rush to use nanomaterials for constructing biodevices, the health risk/safety potential of such materials could be overlooked leading to human injury and the inevitable and expensive civil/criminal litigations that can cause irreparable damage to nanobiosensor companies. Cognizance of regulatory rules ("burdens"?) governing nanomaterials is essential for maintaining federal compliance as well as avoiding stiff penalties for violations. The perceived lack of industry enthusiasm with nanobiosensor products might also be due to inadequate guidance from the multiple state and federal agencies (such as Environmental Protection Agency, EPA, Food and Drug Administration, FDA, Occupational Safety and Health Administration, OSHA, etc.) overseeing nanomaterials acquisition and use, in addition to the technological/societal concerns discussed above. In an era of rising healthcare costs, nanobiosensors success in the marketplace will depend on whether cost cuts are enabled, regardless of a sensor's superior technical performance.

Sometimes too, scientific exuberance does not match reality. In the real world, pre-test sample preparation and processing continue to be major roadblocks for launching commercial biosensors. Unprocessed

Corresponding authors: Komandoor Achyuthan, Ph.D., Sandia National Laboratories, Albuquerque, NM 87508, USA, Tel: 505-284-8979; E-mail: kachyut@ sandia.gov

Received February 26, 2011; Accepted March 14, 2011; Published March 16 , 2011

Citation: Achyuthan K (2011) Whither Commercial Nanobiosensors? J Biosens Bioelectron 2:102e. doi:10.4172/2155-6210.1000102e

Copyright: (c) 2011 Achyuthan K. This is an open-access article distributed under the terms of the Creative Commons Attribution License, which permits unrestricted use, distribution, and reproduction in any medium, provided the original author and source are credited. 
samples will be harder for nanobiosensors to handle compared to "conventional" biosensors, such as "raw" blood tests using glucose meters. Currently at least, fabrication processes for nanomaterials are complex, sometimes hazardous and distant from "green manufacturing." It is also important to remember that miniaturization and scaling may not always work to our advantage. Certain detector technologies (for example, piezoelectric) are better suited to micro than to nano devices. Obviously, there are opportunities and challenges in commercializing nanobiosensors and the foregoing simply skims the surface of such a complicated topic, hoping to stimulate further conversations and debate.
In conclusion, it behooves us to be inquisitive, detached, vigilant and skeptical in order to separate "wheat from the chaff" with regard to the commercial potential of nanobiosensors. Otherwise, the outcome may be surprising and likely unpleasant. In this sense, commercializing nanobiosensors is not much different from commercializing any biomedical product, including biosensors. Perhaps in the field of nanobiosensors, it is wise to under-promise the tax payers or the investors/venture capitalists and over-deliver, rather than the other way around! So, "whither commercial nanobiosensors?" With apologies to Yogi Berra: "it is hard to make predictions, especially about the future." 